Mediterránea Ser. Biol. (1990), n.0 12. Pág. 139-148

\title{
ECOLOGÍA de LAS POBLACIONES dE COLÉMBOLOS EDÁFICOS EN UN PRADO Y UN PINAR DE LA REGIÓN SUBMEDITERRÁNEA DE NAVARRA ${ }^{1}$
}

\author{
por \\ J. I. ARBEA ${ }^{2}$ y R. JORDANA ${ }^{2}$
}

\section{RESUMEN}

Se han estudiado las poblaciones de colémbolos de dos comunidades vegetales diferentes (prado y pinar de pino albar) de Navarra. En el prado se han encontrado un total de 32 especies; de ellas, 19 son exclusivas de este medio y han desaparecido en el pinar. El pinar muestra una población de colémbolos original, con 21 especies exclusivas del total de 34 especies presentes.

El análisis de la diversidad y similaridad, así como la comparación entre los biotopos por medio del Análisis Factorial de Correspondencias, muestra una separación neta entre estos dos biotopos, que presentan una gran sustitución de especies. El pinar es más complejo (más rico), pero, sin embargo, es menos estable que el prado (menor uniformidad).

PALABRAS CLAVE: Collembola, prado, pinar, diversidad, Análisis de Correspondencias, similaridad.

\section{SUMMARY}

Ecology of collembolan populations in a prairie and a pine forest soils of submediterranean region of Navarra (Northern Iberian Peninsula). The collembolan populations of two different plant communities (prairie and Scots pine forest) of Navarra (Northern Iberian Peninsula) have been studied. In the prairie soil a total of 32 species have been found; out of them, 19 species are exclusive of this environment, having disappeared in the pine forest. The soil of the pine grove shows an original collembolan population with 21 exclusive species of the total 34 species.

The analysis of Diversity and Similarity, as well as comparisons among biotopes by means of correspondences analysis, show a clear separation between those two biotopes, which shows a high substitution. The pine grove is more complex (it is richer), but, however, it is a less stable biotope than the prairie (the Equitability is smaller).

KEYWORDS: Collembola, prairie, pine forest, diversity, Correspondences analysis, Similarity.

(1) Trabajo realizado dentro del proyecto 0220/81 de la CAICYT.

(2) Departamento de Zoología, Universidad de Navarra, E-31080 Pamplona. 


\section{INTRODUCCIÓN}

El presente trabajo forma parte de un estudio más amplio sobre el efecto de la explotación y repoblación forestal sobre la fauna del suelo. Para dicho estudio se seleccionaron las comunidades vegetales que caracterizan Navarra en sus diferentes regiones bioclimáticas y los biotopos resultantes de las correspondientes alteraciones de las mismas, debidas a la deforestación y repoblación con especies no autóctonas.

En trabajos anteriores, ya hemos puesto de manifiesto el efecto que produce la repoblación con coníferas, en distintas regiones de Navarra, sobre las poblaciones de colémbolos (ARBEA y JORDANA, 1985 a y b, 1987; JORDANA et al., 1987). En el análisis que sigue nos proponemos estudiar las poblaciones de colémbolos en dos comunidades vegetales de la región submediterránea de Navarra: un pinar de Pinus sylvestris y un prado.

\section{MATERIAL Y MÉTODOS}

Los dos biotopos, prado y pinar, se muestrearon estacionalmente durante un año natural completo (primavera, verano y otoño de 1982 e invierno de 1983). Se diferenciaron dos niveles dentro del suelo: $\mathrm{H}$ (capa orgánica) y S (3-5 primeros centímetros de suelo mineral). De cada nivel se tomó una cantidad de un peso comprendido entre 200 y 400 gr. según la profundidad, correspondiendo a una superficie de $25 \times 25 \mathrm{~cm}$. Los microartrópodos fueron extraídos por el método de «Berlese-Tullgren».

En cada ocasión de muestreo se determinaron un conjunto de parámetros fisicoquímicos: temperatura, $\mathrm{pH}$ y humedad en la superficie del suelo y a unos $10 \mathrm{~cm}$. de profundidad. El contenido hídrico del suelo se ha medido por la diferencia de peso entre la muestra fresca (peso húmedo) y una vez desecada a $20^{\circ} \mathrm{C}$ y $40 \%$ de humedad ambiental (peso seco):

$\mathrm{CH}=100$ (Peso fresco-Peso seco)/Peso fresco.

Se han realizado las medidas en estas condiciones debido a que el agua utilizable del suelo es menor que la obtenida por desecado a $105^{\circ} \mathrm{C}$, por lo que nos parece más adecuada la medida obtenida al final del período de extracción, en el que ningún microartrópodo queda en el suelo, a excepción de los posibles estados latentes (animales en criptobiosis o en estado de huevo).

Con el fin de comparar la estructura de la población de colémbolos en los distintos biotopos, hemos utilizado varios índices de diversidad:

Diversidad (SHANNON y WEAVER, 1949): $\mathrm{H}^{\prime}=-\Sigma \mathrm{Pi} \ln \mathrm{Pi}$

Riqueza específica (MARGALEF, 1951): $\mathrm{R}=\mathrm{S}-1 / \mathrm{ln} \mathrm{N}$

Uniformidad (LLOYD y GHELARDI, 1964): $\mathrm{J}=\mathrm{H}^{\prime} / \mathrm{In} \mathrm{S}$

donde $\mathrm{Pi}=$ abundancia de la especie $\mathrm{i}$ en el biotopo considerado; $\mathrm{S}=$ número de especies; $\mathrm{N}=$ número de individuos.

Para determinar los agrupamientos de los biotopos en función de sus perfiles faunísticos, hemos realizado un Análisis Factorial de Correspondencias; este tipo de análisis permite obtener una representación objetiva de un cuadro de contingencia (especies $x$ biotopos), por lo que ha sido ampliamente utilizado en la descripción de las estructuras biocenóticas de los colémbolos edáficos (BONNET et al., 1979; ARBEA y JORDANA, 1985 a y b, 1987; JORDANA et al., 1987). Para la realización de este análisis se ha utilizado el programa ANAFACOR (LAGARDE, 1983) modificado por nosotros. 
Las afinidades entre los biotopos y los agrupamientos que de ellas se derivan se han establecido comparando los biotopos dos a dos y calculando el índice de similaridad de Jaccard: $J=N a b /(\mathrm{Na}+\mathrm{Nb}-\mathrm{Nab})$, donde $\mathrm{Nab}$ es el número de especies comunes a los dos biotopos; $\mathrm{Na}$ y $\mathrm{Nb}$ son el número de especies de cada uno de los biotopos, que se comparan. A partir de la matriz de similaridad entre los biotopos construimos la jerarquía correspondiente que se representa por un dendrograma. Para obtener la jerarquía hemos utilizado el método de la media de Sokal y Michener, 1958 (CUADRAS, 1981).

\section{DESCRIPCIÓN DE LOS BIOTOPOS}

Los dos biotopos estudiados en este trabajo, prado y pinar, se sitúan en la localidad de Bigüezal (U.T.M. 30TXN52) (Navarra), y se encuentran a unos $900 \mathrm{~m}$. de altitud. El clima es Subhúmedo Mesotérmico II, con gran falta de agua en verano. El régimen termo-hídrico estival corresponde a un tipo de clima de transición entre el submediterráneo y el oceánico-mediterráneo (Índice de Vernet $=-2.8$ ).

Se ha estudiado un pinar de Pinus sylvestris de una implantación muy antigua, más de 150 años. Está sustituyendo a un hayedo, pero se encuentra dentro del área propia de distribución de Pinus sylvestris, que es autóctono de la región (FERNÁNDEZ DE LEÓN, 1982). En la zona talada del pinar se ha establecido un prado que se utiliza para pastos, que también ha sido estudiado.

En la figura 1 se representan varias características de los biotopos. $\mathrm{El} \mathrm{pH}$ es neutro en el prado y bastante ácido en el pinar: entre 4 y 5 (fig. 1 A y D).
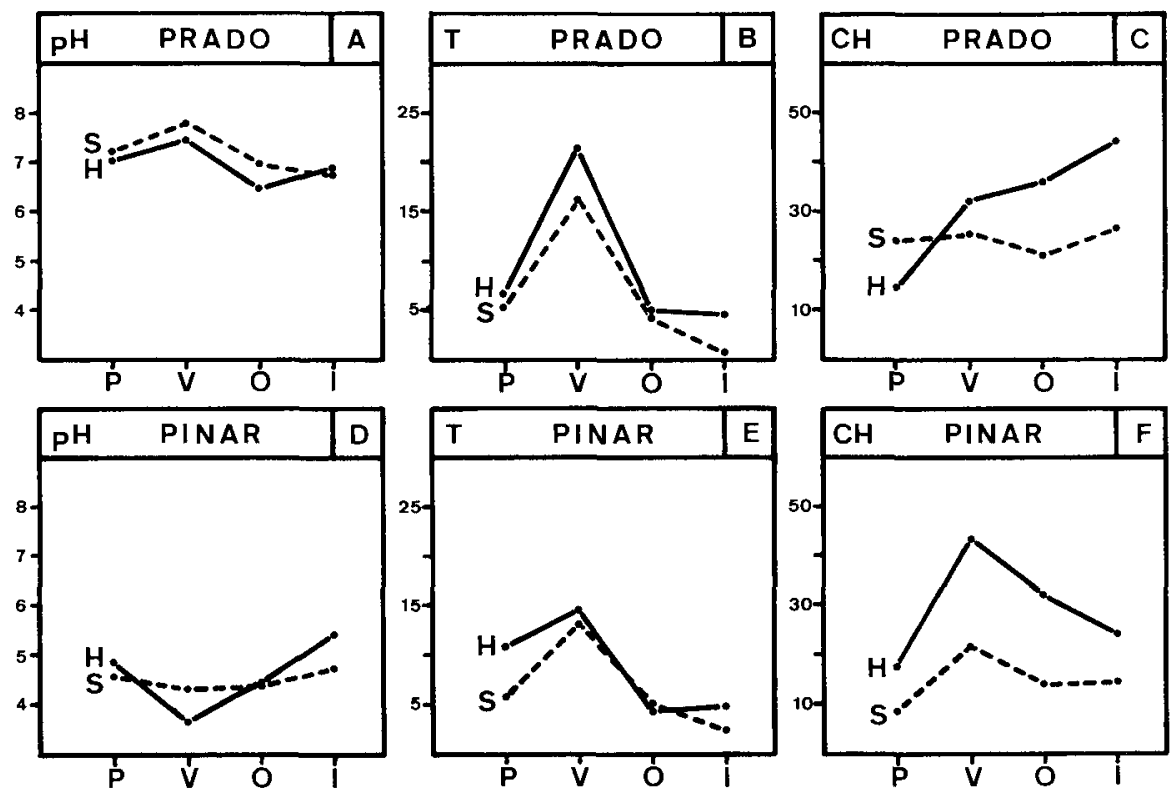

Figura 1.-Variación estacional del $\mathrm{pH}$, de la temperatura $\left(\mathrm{T},{ }^{\circ} \mathrm{C}\right)$ y del contenido hídrico $(\mathrm{CH}, \%)$, en el prado (A, B, C) y en el pinar (D, E, F), por horizontes (capa orgánica: H; suelo mineral: S). P: primavera; V: verano; O: otoño; I: invierno. 
Las temperaturas alcanzan los valores máximos en verano (fig. $1 \mathrm{~B}$ y E). Las oscilaciones térmicas son mayores en el prado, que presenta un máximo de verano mucho más acusado, ya que se trata de un medio abierto sin protección contra la insolación. En el prado, el contenido hídrico supera generalmente el $20 \%$ (fig. $1 \mathrm{C}$ ), por lo que está saturado en agua (POZO et al., 1986). Por el contrario, en el pinar el suelo no sobrepasa el umbral del $20 \%$, aunque las capas orgánicas superficiales, que retienen más el agua, pueden alcanzar humedades relativamente altas (fig. $1 \mathrm{~F}$ ).

\section{COMPOSICIÓN FAUNÍSTICA}

Se ha encontrado un total de 53 especies de colémbolos, de las cuales 32 se encuentran en el prado y 34 en el pinar.

En la tabla I se da el número de ejemplares de cada especie para cada biotopo y estación del año, considerando el conjunto del suelo (niveles $\mathrm{H}$ y S unidos). La novena columna refleja el coeficiente general de frecuencia para cada especie, definido como la relación entre el número de muestras en las que se encuentra una especie y el número total de muestras (ocho en este caso) multiplicado por 100. Además, se indica la categoría morfoecológica a la que pertenece cada especie: $\mathrm{A}$, atmobios; $\mathrm{M}$, hemiedáficos mesófilos; $\mathrm{X}$, hemiedáficos xerófilos; $\mathrm{E}$, euedáficos.

De las 32 especies presentes en el prado, 19 son exclusivas de este medio $y$ han desaparecido en el pinar. Las poblaciones experimentan cambios notables en el número de especies y ejemplares a lo largo del año, debido principalmente a las variaciones que se producen en el nivel superficial $(\mathrm{H})$, ya que el suelo mineral (S) es muy pobre durante todo el año (fig. 2). La población máxima se alcanza en primavera, debido al gran número de ejemplares de Xenylla tullbergi y Folsomia sexoculata encontrados en la capa orgánica superficial $(\mathrm{H})$; estas dos especies son accidentales en este prado y sólo aparecen durante la primavera. El número de especies sigue una evolución paralela al número de ejemplares, con un máximo en primavera y un mínimo estival.

El prado lo podemos definir por nueve especies que presentan una abundancia superior al 5\%: Xenylla tullbergi, Folsomia sexoculata, Isotomurus fucicola, Friesea truncata y Lepidocyrtus lusitanicus, limitadas a la capa orgánica superficial, y Cryptopygus thermophilus, Isotomodes bisetosus y Protaphorura armata, que, aunque viven preferentemente en las capas superficiales, se pueden encontrar también en los primeros centímetros de suelo mineral (fig. 2). En conjunto, estas ocho especies suponen el $89 \%$ del total de ejemplares de este prado. La población de colémbolos está dominada por los elementos euedáficos (fig. 3). Entre ellos destaca el elevado número de especies del género Mesaphorura (cinco especies).

El pinar muestra una población de colémbolos muy original respecto a la del prado. De las 34 especies presentes en este biotopo, 21 son exclusivas. El número de especies y de ejemplares es muy bajo en el suelo (S) durante todo el año; se produce un máximo en verano debido a que algunas especies, como las formas ecomórficas de Hypogastrura acuminata, se resguardan del rigor estival en las capas profundas del suelo; otras especies, como Folsomia sexo- 
TABLA I

\begin{tabular}{|c|c|c|c|c|c|c|c|c|c|c|c|}
\hline & \multirow[b]{2}{*}{$\mathrm{CM}$} & \multicolumn{4}{|c|}{ PINAR } & \multicolumn{4}{|c|}{ PRADO } & \multirow[b]{2}{*}{ Fr. } \\
\hline & & & $\mathbf{P}$ & $\mathrm{v}$ & $\mathrm{o}$ & I & $\mathrm{P}$ & $\mathrm{v}$ & $\mathrm{o}$ & 1 & \\
\hline $\mathrm{HAC}$ & Hypogastrura acuminata ............... & $\mathbf{M}$ & 16 & 4 & 37 & 10 & & & & & 50 \\
\hline CAR & 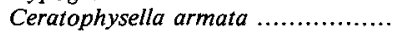 & $\mathbf{M}$ & & 1 & & & & & & & 13 \\
\hline CEN & Ceratophysella engadinensis .......... & $\mathbf{M}$ & 48 & 17 & 6 & 1 & & & & & 50 \\
\hline WAN & Willemia anophthalma ................. & E & & & 29 & & & & & & 13 \\
\hline OD2 & Odontella (Superodontella) $s p . . . . .$. & $\mathbf{M}$ & & & 1 & & & & & & 13 \\
\hline XAR & 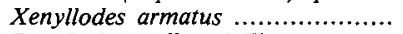 & $\mathrm{E}$ & & & & 2 & & . & & & 13 \\
\hline PAS & Pseudachorutella asigillata ............. & $\mathbf{M}$ & & 16 & I & & & & & & 25 \\
\hline PPA & Pseudachorutes palmiensis ............ & $\mathbf{M}$ & & 46 & 8 & & & & & & 25 \\
\hline MPY & Micranurida pygmaea & $\mathbf{E}$ & & 1 & 1 & & & & & & 25 \\
\hline DMO & 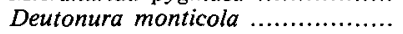 & $\bar{M}$ & & & 1 & 1 & & & & & 25 \\
\hline NMU & Neanura muscorum .......................... & $\mathbf{M}$ & & 1 & & & & & & & 13 \\
\hline $\mathrm{OAB}$ & Oligaphorura absoloni ................... & $\mathrm{E}$ & 4 & 4 & & & & & & & 25 \\
\hline MHY & Mesaphorura hylophila ...................... & $\bar{E}$ & 2 & & & & & & & & 25 \\
\hline IMI & Isotomiella minor $\ldots \ldots \ldots \ldots \ldots \ldots \ldots \ldots \ldots \ldots \ldots \ldots \ldots$ & $\bar{E}$ & 49 & 15 & 1 & 6 & & & & & 50 \\
\hline VAR & Vertagopus arboreus & $X$ & & & & $I$ & & & & & 13 \\
\hline$E A L$ & Entomobrya albocincta $\ldots \ldots \ldots \ldots \ldots$ & A & & 2 & & 2 & & & & & 25 \\
\hline ENI & 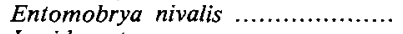 & A & & 4 & & & & & & & 13 \\
\hline LCY & 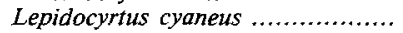 & $\mathrm{M}$ & 2 & & 1 & & & & & & 25 \\
\hline LL1 & 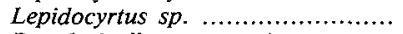 & $\mathbf{M}$ & & & 4 & & & & & & 13 \\
\hline PNA & Pseudosinella navarrensis .............. & $\mathrm{M}$ & & & 1 & & & & & & 13 \\
\hline SNI & Sminthurinus niger ...................... & $\mathrm{x}$ & & & 3 & & & & & & 13 \\
\hline $\mathrm{XSC}$ & Xenylla schillei & $\mathrm{X}$ & 3 & 96 & 5 & 10 & & 1 & & 3 & 75 \\
\hline XTU & Xenylla tullbergi ............... & $\mathrm{x}$ & & 5 & & 2 & 136 & & & & 38 \\
\hline MMA & Mesaphorura macrochaeta ............ & $\mathrm{E}$ & 1 & & 6 & & 5 & & 1 & & 50 \\
\hline $\mathrm{PCA}$ & Paratullbergia callipygos ............... & $\overline{\mathrm{E}}$ & $i$ & & & & 6 & & & & 25 \\
\hline INO & Isotoma (Parisotoma) notabilis ..... & & 25 & 9 & 9 & & 1 & & & 1 & 63 \\
\hline PMO & Pseudisotoma monochaeta ............ & $\mathrm{M}$ & 226 & 190 & 87 & 4 & & & 1 & & 63 \\
\hline FSX & Folsomia sexoculata ........................ & M & 282 & 2378 & 1130 & 28 & 83 & & & & 63 \\
\hline FMA & Folsomia manolachei & $\mathbf{M}$ & & & & 1 & & & 1 & & 25 \\
\hline EST & Entomobrya strigata ..................... & A & & & 9 & 5 & 1 & & & & 38 \\
\hline HMA & Heteromurus major ................... & A & & 13 & 5 & 2 & 6 & & & 2 & 63 \\
\hline PAL & Pseudosinella alba $\ldots \ldots \ldots \ldots \ldots \ldots \ldots$ & $\mathrm{E}$ & & 2 & & & 1 & & & & 25 \\
\hline TMI & Tomocerus minor & A & & & 1 & & 1 & & & & 25 \\
\hline SAU & 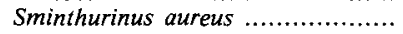 & $\mathrm{M}$ & 1 & & 2 & & & & 1 & & 38 \\
\hline PGU & Pseudachorutes guadalajarensis ..... & $\mathrm{M}$ & & & & & & 1 & & 1 & 25 \\
\hline FTR & Friesea truncata & $\mathbf{M}$ & & & & & 7 & & 21 & 11 & 38 \\
\hline BPA & Brachystomella parvula ................ & M & & & & & 2 & & & 6 & 25 \\
\hline PAR & Protaphorura armata ......... & $\mathrm{E}$ & & & & & 5 & & 34 & 7 & 38 \\
\hline MAF & Metaphorura affinis ................ & $\mathrm{E}$ & & & & & 4 & 4 & 1 & & 38 \\
\hline MCR & 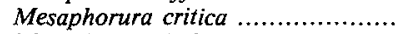 & $\bar{E}$ & & & & & 2 & & & & 13 \\
\hline MIT & Mesaphorura italica .................... & $\mathrm{E}$ & & & & & & 2 & & & 13 \\
\hline MKR & Mesaphorura krausbaueri ............. & $\bar{E}$ & & & & & 3 & & & & 13 \\
\hline MYO & Mesaphorura yosii ............ & $\mathrm{E}$ & & & & & 2 & & & & 13 \\
\hline IBI & Isotomodes bisetosus ........ & $\overline{\mathrm{E}}$ & & & & & 41 & 15 & 1 & & 38 \\
\hline $\mathrm{CTH}$ & Cryptopygus thermophilus & $x$ & & & & & & 29 & 25 & 102 & 38 \\
\hline IFU & 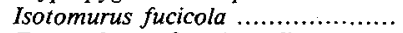 & A & & & & & 3 & & 20 & 43 & 38 \\
\hline EQU & Entomobrya cf. quinquelineata ...... & A & & & & & & & 1 & & 13 \\
\hline LLU & Lepidocyrtus lusitanicus .................. & M & & & & & 17 & & 18 & 2 & 38 \\
\hline PS1 & 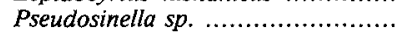 & $\mathrm{E}$ & & & & & 1 & & & & 13 \\
\hline CAL & 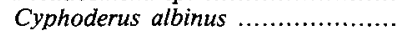 & $\bar{E}$ & & & & & 1 & & & & 13 \\
\hline BPI & Bourletiella pistillum & A & & & & & 6 & & & & 13 \\
\hline BRA & Bourletiella radula ............................. & A & & & & & 8 & & & & 13 \\
\hline SPU & Sphaeridia pumilis ........................ & M & & & & & 1 & & & & 13 \\
\hline & N. TOTAL EJEMPLARES & & 660 & 2804 & 1349 & 75 & 343 & 52 & 125 & 178 & \\
\hline & N. TOTAL DE ESPECIES & & 13 & 18 & 23 & 14 & 24 & 6 & 12 & 10 & \\
\hline & DIVERSIDAD & & 1.45 & 0.70 & 0.78 & 2.06 & 1.96 & 1.16 & 1.82 & 1.30 & \\
\hline & RIQUEZA & & 1.85 & 2.14 & 3.05 & 3.01 & 3.94 & 1.27 & 2.28 & 1.74 & \\
\hline & UNIFORMIDAD & & 0.56 & 0.24 & 0.25 & 0.78 & 0.62 & 0.65 & 0.73 & 0.57 & \\
\hline
\end{tabular}

TABLA I.-Número de ejemplares por unidad de muestreo y valores de la diversidad en los distintos «Biotopos-Estación». Coeficiente general de frecuencia (Fr.) de las especies. Categorías morfoecológicas: $\mathrm{CM}(\mathrm{M}$, hemiedáficos mesófilos; $\mathrm{X}$, hemiedáficos xerófilos; $\mathrm{E}$, euedáficos; $\mathrm{A}$, atmobios). $\mathrm{P}$ : primavera; V: verano; $\mathrm{O}$ : otoño; $\mathrm{I}$ : invierno. 
culata y Pseudisotoma monochaeta, sufren una explosión demográfica en las capas superficiales del suelo durante el verano, por lo que pueden encontrarse accidentalmente en profundidad. En la capa orgánica superficial $(\mathrm{H})$, tanto el número de especies como el número de ejemplares aumenta progresivamente de primavera a otoño (fig. 2). La población tiene dos máximos, en verano y otoño, debidos a la elevada dominancia de Folsomia sexoculata.

Dos especies, que suponen el $88 \%$ del total de ejemplares, definen el pinar: Folsomia sexoculata y Pseudisotoma monochaeta. Aunque con una abundancia menor, otras especies importantes en el pinar por su elevada frecuencia son Xenylla schillei, Ceratophysella engadinensis, Isotomiella minor e Hypogastrura acuminata. Todas estas especies son características de la hojarasca y raramente se encuentran a mayor profundidad (fig. 2). Respecto al prado, aumentan los elementos mesófilos, mientras que disminuyen los atmobios y euedáficos (fig. 3). Esto se debe fundamentalmente al gran número de Neanúridos que son exclusivos de este pinar, como Pseudachorutella asigillata, Pseudachorutes palmiensis, Deutonura monticola, Neanura muscorum, que son especies hemiedáficas mesófilas típicamente forestales.
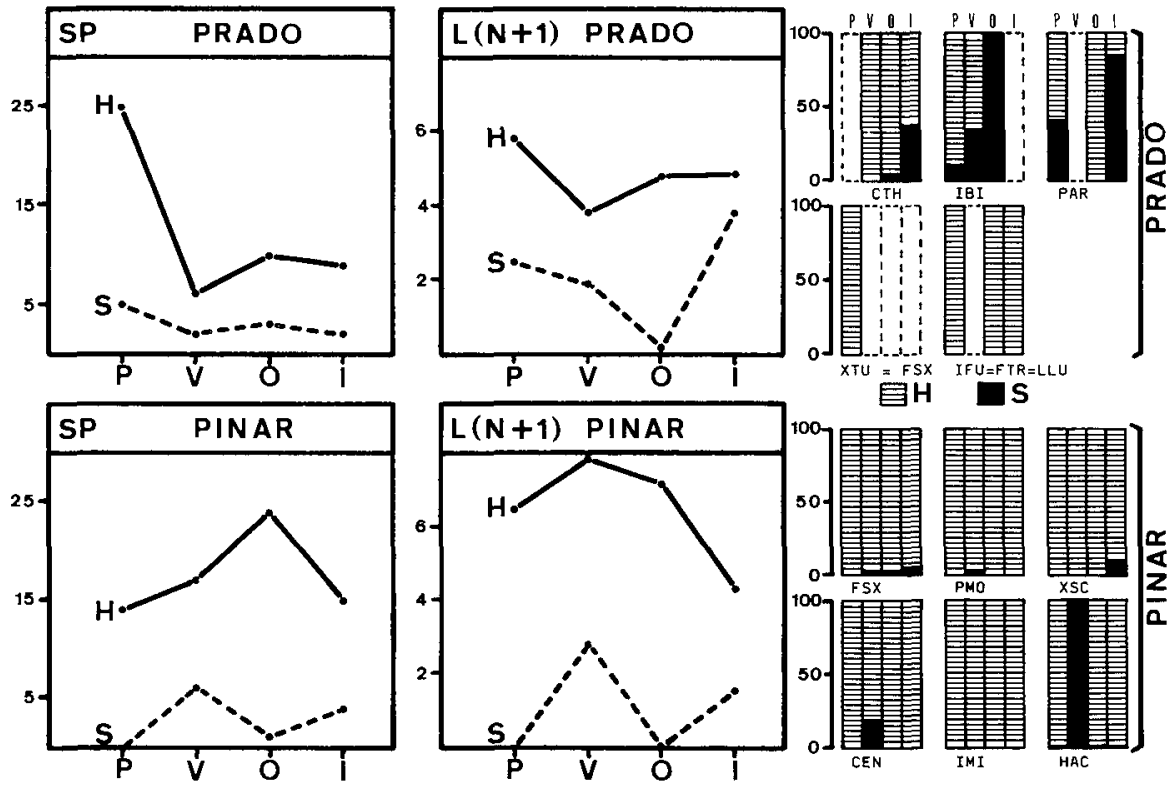

Figura 2.-Variación estacional, en cada horizonte del suelo (capa orgánica: $\mathrm{H}$; suelo mineral: $\mathrm{S}$ ), del número de especies (SP), del número total de ejemplares (N) y de las especies más abundantes y frecuentes en cada uno de los biotopos. $\mathrm{P}$ : primavera; $\mathrm{V}$ : verano; $\mathrm{O}$ : otoño; I: Invierno. CTH: C. thermophilus; XTU: $X$. tullbergi; FSX: $F$. sexoculata; IFU: $I$. fucicola; IBI: I. bisetosus; PAR: P. armata; FTR: F. truncata; LLU: L. lusitanicus; PMO: $P$. monochaeta; XSC: $X$. schillei; CEN: C. engadinensis; IMI: $I$. minor; HAC: $H$. acuminata. 

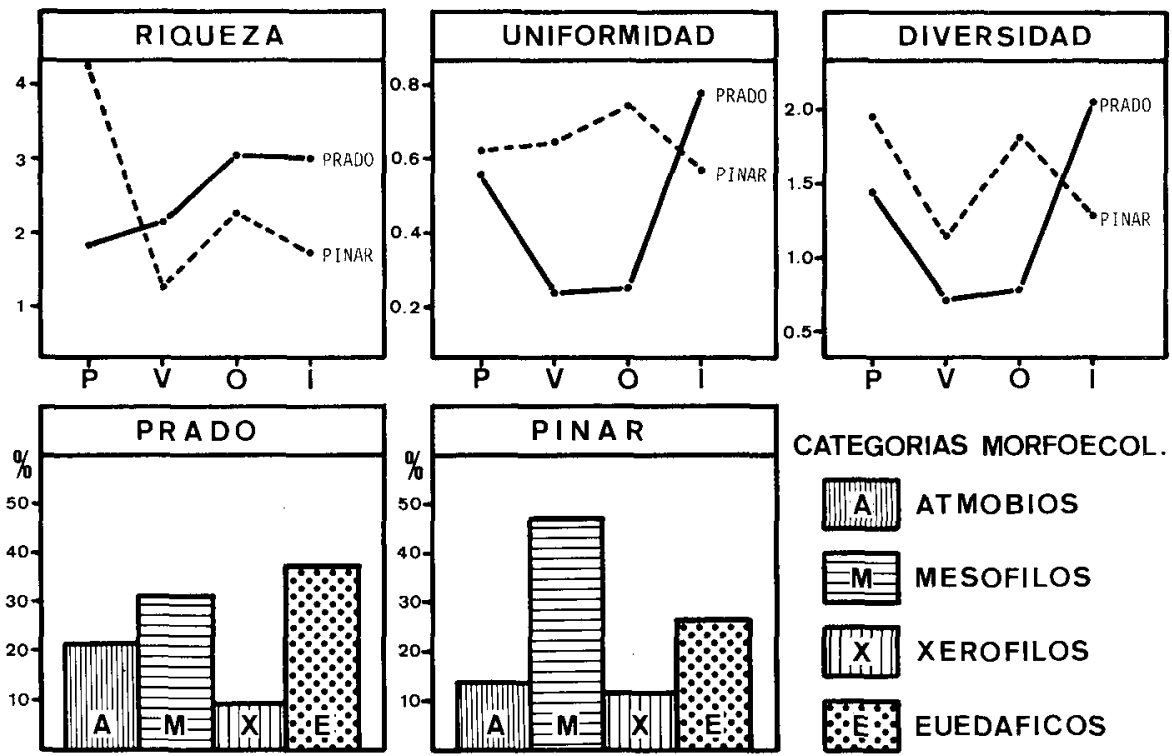

CATEGORIAS MORFOECOL.

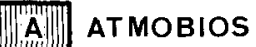

M目 MESOFILOS

[X] XEROFILOS

E: EUEDAFICOS

Figura 3.-Variación estacional de la riqueza, uniformidad y diversidad en el prado y en el pinar. Porcentaje de especies pertenecientes a las diferentes categorías morfoecológicas. P: primavera; V: verano; O: otoño; I: invierno.

\section{ESTRUCTURA DE LAS POBLACIONES}

\section{Diversidad, riqueza y uniformidad}

En la figura 3 se representa la variación estacional de la diversidad y de sus dos componentes, riqueza y uniformidad, en el prado y en el pinar. Consideramos aquí las poblaciones globales de colémbolos edáficos, sin tener en cuenta los distintos niveles del suelo, ya que la capa de suelo mineral es muy pobre en especies en ambos biotopos (seis especies como máximo), por lo que obtendríamos valores de diversidad poco significativos.

Excepto en primavera, el prado presenta una riqueza inferior a la del pinar. En el prado la riqueza varía considerablemente a lo largo del año, dándose los mínimos en verano e invierno; no obstante, es un medio con una uniformidad elevada y relativamente constante. Por lo tanto, en términos de diversidad, el prado presenta una estructura más equilibrada, especialmente en primavera y otoño (mayor diversidad).

El pinar se puede considerar más complejo que el prado (mayor riqueza), pero menos estable (menor uniformidad), especialmente en verano y otoño. En estas estaciones el pinar presenta una estructura más desequilibrada debido a la elevada dominancia de Folsomia sexoculata. 


\section{Resultados del Análisis Factorial de Correspondencias}

Este análisis se ha realizado con los ocho «Biotopos-Estación» y las 33 especies de colémbolos más importantes, y los resultados se representan en la fig. 4. Los dos primeros ejes explican casi el $60 \%$ del total de la varianza, por lo que son suficientes para reflejar la estructura biocenótica de las poblaciones de colémbolos.

El eje 1 (43.9\% de la varianza explicada) separa el prado (valores positivos) del pinar (valores negativos). El eje 2 ( $15.9 \%$ de varianza) establece un gradiente estacional muy acusado dentro del prado, enfrentando el verano (valores positivos) a otoño-invierno (valores negativos).

Las especies ecológicamente neutras aparecen agrupadas alrededor del origen. Solamente dos especies, Mesaphorura macrochaeta (MMA) y Heteromu-

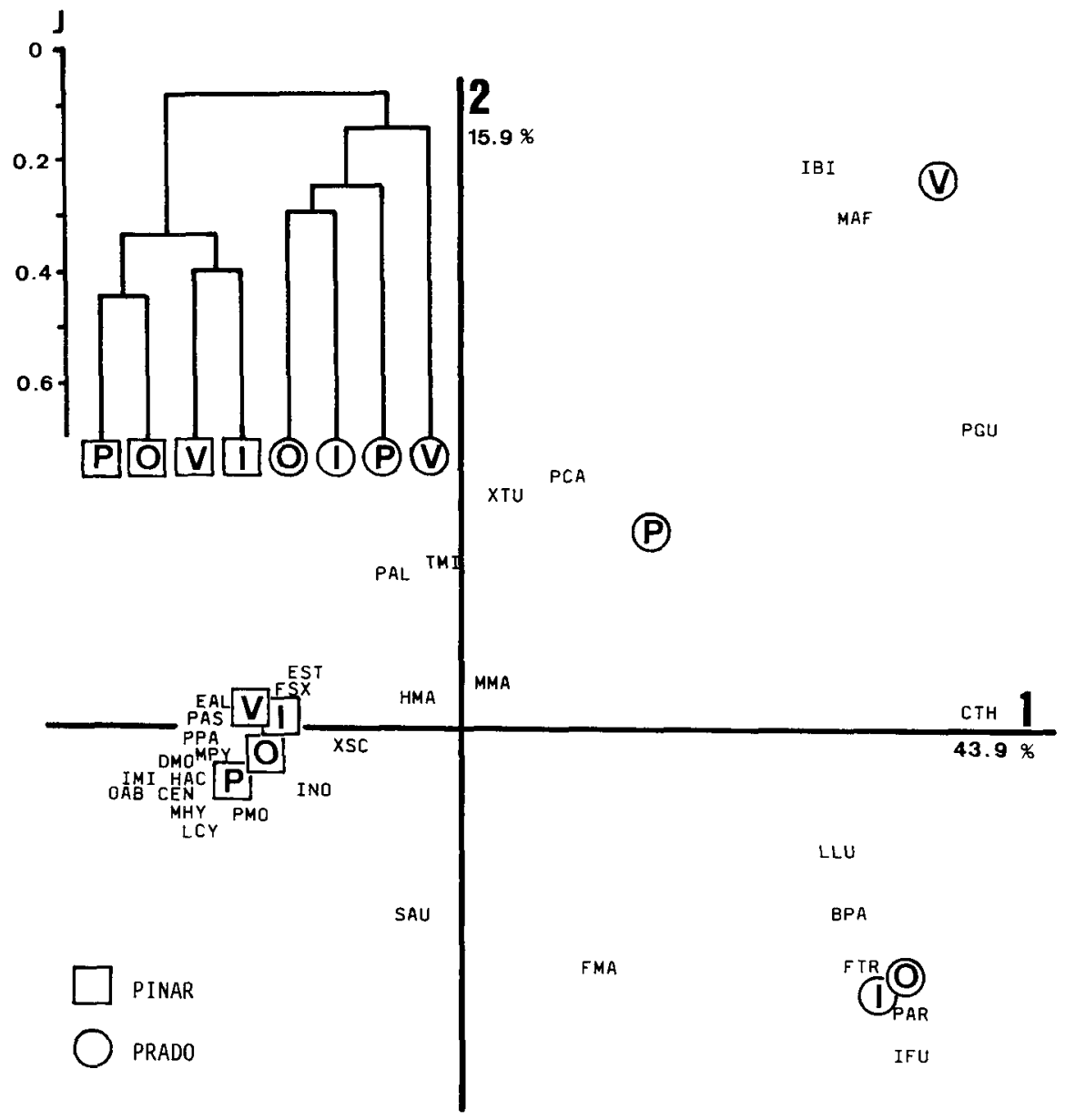

Figura 4.-Análisis Factorial de Correspondencias de los ocho «Biotopos-Estación». Proyección según los dos primeros ejes. Dendrograma correspondiente a la clasificación jerárquica de los ocho «Biotopos-Estación» según el índice de similaridad de Jaccard. P: primavera; V: verano; O: otoño; I: invierno. Abreviaturas de las especies según la Tabla I. 
rus major (HMA), aparecen bien representadas en los dos biotopos, aunque con una abundancia baja.

Un segundo conjunto de especies aparece formando una nube de puntos situada en los valores positivos del eje 1, y alargándose según el eje 2 . Se corresponde con las especies exclusivas del prado: Pseudachorutes guadalajarensis (PGU), Friesea truncata (FTR), Brachystomella parvula (BPA), Protaphorura armata (PAR), Metaphorura affinis (MAF), Isotomodes bisetosus (IBI), Crytopygus thermophilus (CTH), Isotomurus fucicola (IFU), Lepidocyrtus lusitanicus (LLU).

El tercer conjunto de especies se sitúa en los valores negativos del eje 1 . Se identifica con el pinar, y comprende 11 especies exclusivas de este biotopo: Hypogastrura acuminata (HAC), Ceratophysella engadinensis (CEN), Pseudachorutella asigillata (PAS), Pseudachorutes palmiensis (PPA), Micranurida pygmaea (MPY), Deutonura monticola (DMO), Oligaphorura absoloni (OAB), Mesaphorura hylophila (MHY), Isotomiella minor (IMI), Entomobrya albocincta (EAL), Lepidocyrtus cyaneus (LCY). Podemos añadir otras especies que, aunque pueden aparecer en el prado de forma aislada, se encuentran mucho mejor representadas en el pinar: Xenylla schillei (XSC), Isotoma (Parisotoma) notabilis (INO), Pseudisotoma monochaeta (PMO), Folsomia sexoculata (FSX), Entomobrya strigata (EST).

\section{Clasificación jerárquica}

En la figura 4 se representa el dendrograma correspondiente a la clasificación jerárquica de los ocho «Biotopos-Estación», construida en base al índice de similaridad de Jaccard.

Se observan dos agrupamientos, que se identifican con el prado y el pinar. Estos dos biotopos se presentan, por lo tanto, como dos medios distintos, claramente diferenciables por su composición faunística. Las similaridades entre las diferentes estaciones del pinar son mayores que las existentes entre las estaciones del prado. Esto nos está indicando las grandes variaciones estacionales que se producen en el prado, como ya quedaba reflejado en el Análisis Factorial de Correspondencias.

\section{DISCUSIÓN Y CONCLUSIONES}

El estudio de las poblaciones de colémbolos en un prado y un pinar de la región submediterránea de Navarra nos revela la presencia de 53 especies, de las cuales solamente 13 son comunes a los dos biotopos, que serán, por lo tanto, muy diferentes en composición faunística. La especie más abundante es diferente en cada caso: Folsomia sexoculata, típicamente forestal, en el pinar; Cryptopygus thermophilus, característica de medios abiertos, en el prado.

El prado es un biotopo con unas variaciones estacionales muy marcadas y las poblaciones de colémbolos presentan un mínimo muy acusado en verano, como corresponde a un medio abierto con clima de influencia mediterránea. Por el contrario, en el pinar las variaciones estacionales son menos importantes e incluso se da un máximo en verano debido a la explosión demográfica de Folsomia sexoculata. Este comportamiento diferente de los dos biotopos 
se refleja en el hecho de que Mesaphorura macrochaeta se encuentra formando poblaciones bisexuales en el prado, mientras que en el pinar las poblaciones están constituidas sólo por hembras. Esto está de acuerdo con la idea de PETERSEN (1978) de que la bisexualidad en las Mesaphorura es una adaptación a medios impredectibles, sujetos a mayores oscilaciones de las condiciones ambientales; como ocurre, en nuestro caso, en el prado frente al pinar.

\section{BIBLIOGRAFÍA}

ARBEA, J. I. y JORDANA, R. (1985 a). Estudio ecológico de la colembofauna de los suelos del macizo de Quinto Real (Pirineos Occidentales) y descripción de dos especies nuevas: $A$ nurida flagellata sp. n. y Onychiurus subedinensis sp. n. (Insecta, Collembola). Boletin Estación Central de Ecología, 28: 57-80.

ARBEA, J. I. y JORDANA, R. (1985 b). Efecto de una repoblación con coníferas en un robledal de Navarra sobre los colémbolos edáficos. Bolm. Soc. port. Ent., Supl. 1, vol. 2: 277-286.

ARBEA, J. I. y JORDANA, R. (1987). Efecto de la repoblación y explotación forestal en la zona norte de Navarra sobre las poblaciones de colémbolos edáficos. Actas VIII Bienal R. Soc. Española Hist. Nat., I Reunión Biología Ecología del Suelo, Pamplona: 507-515.

BONNET, L., CASSAGNAU, P. et DEHARVENG, L. (1979). Recherche d'une méthodologie dans l'analyse de la rupture des équilibres biocénotiques: applications aux Collemboles édaphiques des Pyrénées. Rev. Ecol. Biol. Sol, 16(3): 373-401.

CUADRAS, C. M. (1981). Métodos de análisis multivariante. Eunibar. Barcelona, 642 pp.

FERNÁNDEZ DE LEÓN, C. (1982). Estudio fanerogámico de la Foz de Arbayún y sierra de Leyre. Tesis de Licenciatura, Universidad de Navarra.

JORDANA, R., ARBEA, J. I., MORAZA, L., MONTENEGRO, E., MATEO, M. D., HERNÁNDEZ, M. A. and HERRERA, L. (1987). Effect of reafforestation by conifers in natural biotopes of middle and South Navarra (Northern Spain). Revue suisse Zool., 94(3): 491-502.

LAGARDE, J. DE (1983). Initiation à l'analyse des données. Dunod. París, 158 pp.

PETERSEN, H. (1978). Sex-ratios and the extent of parthenogenetic reproduction in some collembolan populations. First Int. Sem. Apterygota, Siena: 19-35.

POZO, J., SELGA, D. and SIMON, J. C. (1986). Studies on the Collembolan populations of several plant communities of the Basque Country (Spain). Rev. Ecol. Biol. Sol, 23(2): 215-232. 